\title{
Optimal harvesting from interacting populations in a stochastic environment
}

\author{
EDWARD LUNGU ${ }^{1}$ and BERNT ØKSENDAL ${ }^{2,3}$ \\ ${ }^{1}$ Department of Mathematics, University of Botswana, P.B. 0022 Gaborone, Botswana. \\ E-mail:lungu@mopipi.ub.bw \\ ${ }^{2}$ Department of Mathematics, University of Oslo, Box 1053 Blindern, N-0316 Oslo, Norway. \\ E-mail: oksendal@math.uio.no \\ ${ }^{3}$ Norwegian School of Economics and Business Administration, Helleveien 30, N-5045 Bergen, \\ Norway
}

Consider $n$ populations whose sizes are given by stochastic differential equations driven by $m$ dimensional Brownian motion. We study the following problem: what harvesting strategy from the $n$ populations maximizes the expected total income from the harvest? We formulate this as a (singular) stochastic control problem and give sufficient conditions for the existence of an optimal strategy. Our results lead to the one-at-a-time principle that it is almost surely never optimal to harvest from more than one population at a time.

Keywords: one-at-a-time principle; optimal harvesting; singular stochastic control; stochastic systems; variational inequalities; verification theorem

\section{Introduction}

When two species coexist they compete for a limited supply of food and water. The conditions under which stable coexistence obtains are of the utmost ecological importance. Investigations by Gause and Witt (1935) on the basis of the Volterra model with Verhulst term led to the conclusion that a stable coexistence of two species is possible if the interaction of each species is more inhibitive of its own kind than it is of the other species. This conclusion is true in the case where populations roam freely. For example, in Botswana human settlements are protected from migratory herds of wildlife, and wildlife and livestock are kept apart by means of fences, game reserves and protected areas.

Wildlife populations now live in restricted habitats which, because of Botswana's semiarid climatic conditions, are now subject to highly variable vegetation conditions and lack of water due to frequent droughts. These factors ultimately affect the carrying capacity of these areas and hence the animal populations. It is, therefore, imperative that animal populations should be controlled in order to ensure survival of all the species and the quality of the environment. It is against this background that we develop optimal harvesting models for interacting populations in a stochastic environment in the hope that sustainable wildlife management policies can be developed based on research findings like ours.

The mathematical model introduced and studied in this paper may be regarded as a 
multidimensional generalization of the one-dimensional model studied in Lungu and Øksendal (1997); see also the related paper of Alvarez and Shepp (1998). The sizes of the $n$ interacting populations are modelled as solutions of a (coupled) system of $n$ stochastic differential equations. The harvesting strategy is then introduced as a stochastic control. If the prices per unit of each of the populations are given, the problem is to find a harvesting strategy which maximizes the expected total discounted income from the harvesting, up to the time of extinction of one of the populations. As is clear from our model, we do not exclude extinction, but after extinction there is no more harvesting and therefore it turns out that the optimal harvesting strategy - the strategy which maximizes the expected total time - is in fact usually of a sustainable type; see, for example, the explicit solutions in the corresponding one-dimensional cases studied by Alvarez and Shepp (1998) and Lungu and Øksendal (1997). We use stochastic calculus to give sufficient conditions for the existence of an optimal strategy. The conditions can be used to find the optimal strategy in explicit cases. This is illustrated by means of an example. This example might be more relevant for finance (optimal dividend strategy) than for population applications.

For other results on optimal harvesting in random environments, see Braumann (2000).

Our general results indicate that typically (generically) it will not be optimal to harvest from more than one population at a time (the 'one-at-a-time principle'). There seems to be support for such a conclusion from observations of the behaviour of certain predators. For example, it is observed that a pride of lions will hunt one type of prey at a time. The lions do this because their hunting techniques for various species are not the same - that for the buffalo is different from that for the zebra. The young members of the pride are therefore introduced to one hunting technique at a time. This approach reduces the risk of injury or even death for these young members. The lions are forced to change from hunting one species to another as the population of a species being hunted declines and the probability of a successful hunt reduces. In doing so, however, nature has instilled in the lions an optimal harvesting strategy which ensures that all prey populations are maintained at acceptable levels.

\section{The model}

Suppose that when we do not intervene the sizes or densities $X_{i}(t), 1 \leqslant i \leqslant n$, of interacting populations at time $t$ are given as the strong solutions of $n$ stochastic differential equations

$$
\begin{aligned}
\mathrm{d} X_{i}(t) & =b_{i}(t, X(t)) \mathrm{d} t+\sum_{j=1}^{m} \sigma_{i j}(t, X(t)) \mathrm{d} B_{j}(t) ; \quad s \leqslant t \leqslant T, \\
X_{i}(s) & =x_{i} \in \mathbb{R},
\end{aligned}
$$

where $B(t)=B(t, \omega)=\left(B_{1}(t, \omega), \ldots, B_{m}(t, \omega)\right), t \geqslant 0, \omega \in \Omega$, is $m$-dimensional Brownian motion, and $X(t)=\left(X_{1}(t), \ldots, X_{n}(t)\right), b_{i}$ and $\sigma_{i j}$ are given continuous functions. We assume that $T=T(\omega)$ has the form

$$
T=\inf \{t>0 ;(t, X(t)) \notin S\}
$$


where $S \subset \mathbb{R}^{n+1}$ is a given Borel set with the property that $S \subset\left(\overline{S^{0}}\right)$, with $S^{0}$ being the interior of $S$, and $\left(\overline{S^{0}}\right)$ its closure. We may think of $S$ as the survival set of our population and $T$ as the time of extinction. An ( $n$-dimensional) harvesting strategy is a stochastic process $\gamma(t, \omega)=\left(\gamma_{1}(t, \omega), \ldots, \gamma_{n}(t, \omega)\right) \in \mathbb{R}^{n}, t \geqslant s, \omega \in \Omega$, satisfying the following conditions:

1. $\gamma(t)$ is measurable with respect to the $\sigma$-algebra $\mathscr{F}_{t}$ generated by $B(s, \cdot), s \leqslant t$ (i.e. $\{\gamma(t)\}_{t \geqslant 0}$ is adapted).

2. $\gamma_{i}(t, \omega)$ is non-decreasing with respect to $t$, for almost all (a.a.) $\omega$, for $1 \leqslant i \leqslant n$.

3. $\gamma(t, \omega)$ is right-continuous as a function of $t$ for a.a. $\omega$.

4. $\gamma(s, \omega)=0$ for a.a. $\omega$.

The component $\gamma_{i}(t, \omega)$ of $\gamma(t, \omega)$ represents the total amount harvested from the initial time $s$ up to time $t$ from population number $i, 1 \leqslant i \leqslant n$. If we apply the harvesting strategy $\gamma(t, \omega)$ then the corresponding population vector $X^{\gamma}(t)=\left(X_{1}^{\gamma}(t), \ldots, X_{n}^{\gamma}(t)\right)^{\mathrm{T}}$ satisfies the (matrix) equation

$$
\begin{aligned}
& \mathrm{d} X^{\gamma}(t)=b\left(t, X^{\gamma}(t)\right) \mathrm{d} t+\sigma\left(t, X^{\gamma}(t)\right) \mathrm{d} B(t)-\mathrm{d} \gamma(t), \quad t \geqslant s, \\
& X^{\gamma}\left(s^{-}\right)=\left(x_{1}, \ldots, x_{n}\right)^{\mathrm{T}},
\end{aligned}
$$

where

$$
b=\left(b_{1}, \ldots, b_{n}\right)^{\mathrm{T}}, \quad \sigma=\left[\begin{array}{ccc}
\sigma_{11} & \cdots & \sigma_{1 m} \\
\vdots & & \vdots \\
\sigma_{n 1} & \cdots & \sigma_{n m}
\end{array}\right]
$$

and $(\cdot)^{\mathrm{T}}$ denotes matrix transposition. We define $\Gamma$ to be the set of all harvesting strategies $\gamma$ such that (2.3) has a unique strong solution $X^{\gamma}(t)$ which does not explode before time $T$ and such that $X^{\gamma}(T) \in \bar{S}$. Note the difference between $X^{\gamma}(s)$ and $X^{\gamma}\left(s^{-}\right): X^{\gamma}\left(s^{-}\right)$is the state before harvesting starts at time $t=s$, while $X^{\gamma}(s)$ is the state immediately after. If $\gamma$ consists of an immediate harvest of size $\Delta \gamma$ at time $t=s$, then

$$
X(s)=X\left(s^{-}\right)-\Delta \gamma .
$$

Suppose that the prices/utilities per unit of population number $i$ when harvested at time $t$ are given by $n$ continuous, non-negative functions $f_{i}(t), i=1, \ldots, n$.

Note that in this paper we assume that the prices $f_{i}(t)$ depend on time $t$ only. The density-dependent case, with $f_{i}=f_{i}(t, x)$, opens up a new type of optimal strategies, the so-called 'chattering' variety. This case is studied in Alvarez et al. (2001). For a discussion of density-dependent prices in the one-dimensional situation, see Alvarez (2000).

The total expected discounted utility harvested from time $s$ to time $T$ is given by

$$
J^{\gamma}(s, x)=\mathrm{E}^{s, x}\left[\int_{[s, T]} f(t) \cdot \mathrm{d} \gamma(t)\right],
$$

where $f=\left(f_{1}, \ldots, f_{n}\right)$ and $f \cdot \mathrm{d} \gamma=\sum_{i=1}^{n} f_{i} \cdot \mathrm{d} \gamma_{i}$, and $\mathrm{E}^{s, x}$ denotes expectation with respect to the probability law $Q^{s, x}$ of $Y^{s, x}(t)=\left(t, X^{\gamma}(t)\right)$ for $t \geqslant s$, assuming that $Y^{s, x}\left(s^{-}\right)=(s, x)$. 
Then the optimal harvesting problem is to find the value function $\Phi(s, x)$ and an optimal harvesting strategy $\gamma^{*}(t)$ such that

$$
\Phi(s, x)=\sup _{\gamma \in \Gamma} J^{\gamma}(s, x)=J^{\gamma^{*}}(s, x) .
$$

Remark. Note that we can also give this problem an economic interpretation: we can let $X_{i}(t)$ denote the value at time $t$ of asset/security/investment $i$ and we let $\gamma_{i}(t)$ represent the total amount paid in dividends up to time $t$ from asset $i$. Then $S$ can be interpreted as the solvency set, $T$ as the time of bankruptcy and (2.5) becomes the problem of finding the optimal stream of dividends from this collection of assets. In Jeanblanc-Picqué and Shiryaev (1995) this interpretation is used in a study of a one-dimensional version of (2.5).

In the following we let $s \leqslant t_{1}<t_{2}<\ldots$ denote the jumping times of a given strategy $\gamma \in \Gamma$, and we let $\Delta \gamma\left(t_{k}\right)=\gamma\left(t_{k}\right)-\gamma\left(t_{k}^{-}\right)$be the jump of $\gamma(t)$ at $t=t_{k}$. We let $\gamma^{c}(t):=$ $\gamma(t)-\sum_{s \leqslant t_{k} \leqslant t} \Delta \gamma\left(t_{k}\right)$ be the continuous part of $\gamma(t)$. We now formulate a sufficient condition for a given function $\phi(s, x)$ to be the value function $\Phi(s, x)$ of $(2.5)$ and for a given strategy $\hat{\gamma} \in \Gamma$ to be optimal. See Haussmann and Suo (1995a; 1995b) and Benth and Reikvam (1998) for a viscosity formulation, and see Benth and Reikvam (1998) and Myhre (1998), as well as the references therein, for a connection between (2.5) and an associated optimal stopping problem.

Theorem 2.1. (i) Suppose $\phi \geqslant 0$ is a continuous function on $\bar{S}$, twice continuously differentiable on $S^{0}$, with the following properties:

$$
\begin{gathered}
\frac{\partial \phi}{\partial x_{i}} \geqslant f_{i} \quad \text { on } S^{0}, \text { for all } i=1, \ldots, n \\
L \phi(t, x):=\frac{\partial \phi}{\partial t}+\sum_{i=1}^{n} b_{i}(t, x) \frac{\partial \phi}{\partial x_{i}}+\frac{1}{2} \sum_{i, j=1}^{n}\left(\sigma \sigma^{\mathrm{T}}\right)_{i j}(t, x) \frac{\partial^{2} \phi}{\partial x_{i} \partial x_{j}} \leqslant 0 \quad \text { on } S^{0} .
\end{gathered}
$$

Then

$$
\phi(s, x) \geqslant \Phi(s, x) \quad \text { on } S .
$$

(ii) Define the non-intervention region

$$
D=\left\{(t, x) \in S^{0}, t>s, \text { and } \frac{\partial \phi}{\partial x_{i}}(t, x)>f_{i}(t), \text { for all } i=1, \ldots, n\right\}
$$

Suppose that

$$
L \phi=0 \quad \text { in } D
$$

and that there exists a harvesting strategy $\hat{\gamma}$ such that the following hold:

$$
\begin{gathered}
\left(t, X^{\hat{\gamma}}(t)\right) \in \bar{D} \quad \text { for all } t>s \\
\left(\frac{\partial \phi}{\partial x_{i}}\left(t, X^{\hat{\gamma}}(t)\right)-f_{i}(t)\right) \mathrm{d} \hat{\gamma}_{i}^{c}(t)=0 \quad \text { for all } i=1, \ldots, n
\end{gathered}
$$


(i.e., $\hat{\gamma}_{i}^{c}$ increases only when $\partial \phi / \partial x_{i}=f_{i}$ );

$$
\Delta \phi\left(t_{k}\right):=\phi\left(t_{k}, X^{\hat{\gamma}}\left(t_{k}\right)\right)-\phi\left(t_{k}, X^{\hat{\gamma}}\left(t_{k}^{-}\right)\right)=-f\left(t_{k}\right) \cdot \Delta \hat{\gamma}\left(t_{k}\right)
$$

at all jumping times $t_{k} \geqslant s$ of $\hat{\gamma}\left(t_{k}\right)$; and

$$
\mathrm{E}^{s, x}\left[\phi\left(T_{R}, X^{\hat{\gamma}}\left(T_{R}\right)\right)\right] \rightarrow 0 \quad \text { as } R \rightarrow \infty,
$$

where

$$
T_{R}=T \wedge R \wedge \inf \left\{t>s ;\left|X^{\hat{\gamma}}(t)\right| \geqslant R\right\}
$$

Then

$$
\phi(s, x)=\Phi(s, x) \quad \text { for all }(s, x) \in S
$$

and

$$
\gamma^{*}:=\hat{\gamma} \text { is an optimal harvesting strategy. }
$$

Proof. (i) Choose $\gamma \in \Gamma$ and assume that $\phi \in C^{2}$ satisfies (2.6)-(2.7). Then by Itô's formula for semimartingales (see Protter 1990, Theorem II.7.33),

$$
\begin{aligned}
\mathrm{E}^{s, x} & {\left[\phi\left(T_{R}, X^{\gamma}\left(T_{R}\right)\right)\right]=\mathrm{E}^{s, x}\left[\phi\left(s, X^{\gamma}(s)\right]\right.} \\
& +\mathrm{E}^{s, x}\left[\int_{s^{+}}^{T_{R}} \frac{\partial \phi}{\partial t}\left(t, X^{\gamma}(t)\right) \mathrm{d} t+\int_{s^{+}}^{T_{R}}\left(\frac{\partial \phi}{\partial x_{1}}\left(t, X^{\gamma}\left(t^{-}\right)\right) \mathrm{d} X_{1}^{\gamma}(t)+\ldots+\frac{\partial \phi}{\partial x_{n}}\left(t, X^{\gamma}\left(t^{-}\right)\right) \mathrm{d} X_{n}^{\gamma}(t)\right)\right. \\
& +\sum_{i, j=1}^{n} \int_{s^{+}}^{T_{R}} \frac{1}{2}\left(\sigma \sigma^{\mathrm{T}}\right)_{i j}\left(t, X^{\gamma}(t)\right) \frac{\partial^{2} \phi}{\partial x_{i} \partial x_{j}}\left(t, X^{\gamma}(t)\right) \mathrm{d} t \\
& \left.+\sum_{s<t_{k} \leqslant T_{R}}\left\{\phi\left(t_{k}, X^{\gamma}\left(t_{k}\right)\right)-\phi\left(t_{k}, X^{\gamma}\left(t_{k}^{-}\right)\right)-\sum_{i=1}^{n} \frac{\partial \phi}{\partial x_{i}}\left(t_{k}, X^{\gamma}\left(t_{k}^{-}\right)\right) \Delta X_{i}^{\gamma}\left(t_{k}\right)\right\}\right],
\end{aligned}
$$

where the sum is taken over all jumping times $t_{k} \in\left(s, T_{R}\right]$ of $\gamma(t)$ (and $X^{\gamma}(t)$ ). Using (2.7), this gives

$$
\begin{aligned}
& \mathrm{E}^{s, x}\left[\phi\left(T_{R}, X^{\gamma}\left(T_{R}\right)\right)\right]=\mathrm{E}^{s, x}\left[\phi\left(s, X^{\gamma}(s)\right)\right. \\
& \quad+\int_{s^{+}}^{T_{R}} L \phi\left(t, X^{\gamma}(t)\right) \mathrm{d} t-\int_{s^{+}}^{T_{R}}\left\{\sum_{i=1}^{n} \frac{\partial \phi}{\partial x_{i}}\left(t, X^{\gamma}\left(t^{-}\right)\right) \cdot \mathrm{d} \gamma_{i}(t)\right\} \\
& \left.+\sum_{s<t_{k} \leqslant T_{R}}\left\{\phi\left(t_{k}, X^{\gamma}\left(t_{k}\right)\right)-\phi\left(t_{k}, X^{\gamma}\left(t_{k}^{-}\right)\right)+\sum_{i=1}^{n} \frac{\partial \phi}{\partial x_{i}}\left(t_{k}, X^{\gamma}\left(t_{k}^{-}\right)\right) \Delta \gamma_{i}\left(t_{k}\right)\right\}\right] \\
& \leqslant \phi(s, x)-\mathrm{E}^{s, x}\left[\int_{s^{+}}^{T_{R}} \sum_{i=1}^{n} \frac{\partial \phi}{\partial x_{i}}\left(t, X^{\gamma}\left(t^{-}\right)\right) d \gamma_{i}(t)\right]
\end{aligned}
$$




$$
+\mathrm{E}^{s, x}\left[\sum_{s \leqslant t_{k} \leqslant T_{R}}\left\{\Delta \phi\left(t_{k}, X^{\gamma}\left(t_{k}\right)\right)+\sum_{i=1}^{n} \frac{\partial \phi}{\partial x_{i}}\left(t_{k}, X\left(t_{k}^{-}\right)\right) \Delta \gamma_{i}\left(t_{k}\right)\right\}\right]-\mathrm{E}^{s, x}\left[\sum_{i=1}^{n} \frac{\partial \phi}{\partial x_{i}}(s, x) \Delta \gamma_{i}(s)\right],
$$

where

$$
\Delta \phi\left(t_{k}, X^{\gamma}\left(t_{k}\right)\right)=\phi\left(t_{k}, X^{\gamma}\left(t_{k}\right)\right)-\phi\left(t_{k}, X^{\gamma}\left(t_{k}^{-}\right)\right) \quad \text { for } t_{k} \geqslant s .
$$

Let $\gamma^{c}(t)$ denote the continuous part of $\gamma(t)$, that is,

$$
\gamma^{c}(t)=\gamma(t)-\sum_{s \leqslant t_{k} \leqslant t} \Delta \gamma\left(t_{k}\right)
$$

Then we obtain

$$
\mathrm{E}^{s, x}\left[\phi\left(T_{R}, X^{\gamma}\left(T_{R}\right)\right)\right] \leqslant \phi(s, x)-\mathrm{E}^{s, x}\left[\int_{s}^{T_{R}} \sum_{i=1}^{n} \frac{\partial \phi}{\partial x_{i}} \mathrm{~d} \gamma_{i}^{c}\right]+\mathrm{E}^{s, x}\left[\sum_{s \leqslant t_{k} \leqslant T_{R}} \Delta \phi\left(t_{k}, X^{\gamma}\left(t_{k}\right)\right)\right] .
$$

By the mean value property, we have

$$
\Delta \phi\left(t_{k}, X^{\gamma}\left(t_{k}\right)\right)=\sum_{i=1}^{n} \frac{\partial \phi}{\partial x_{i}}\left(t_{k}, \hat{X}_{(k)}^{\gamma}\right) \Delta X_{i}^{\gamma}\left(t_{k}\right)=-\sum_{i=1}^{n} \frac{\partial \phi}{\partial x_{i}}\left(t_{k}, \hat{X}_{(k)}^{\gamma}\right) \Delta \gamma_{i}\left(t_{k}\right)
$$

for some point $\hat{X}_{(k)}^{\gamma}$ on the line connecting the points $X^{\gamma}\left(t_{k}^{-}\right)$and $X^{\gamma}\left(t_{k}\right)$.

Hence

$$
\mathrm{E}^{s, x}\left[\phi\left(T_{R}, X^{\gamma}\left(T_{R}\right)\right)\right] \leqslant \phi(s, x)-\mathrm{E}^{s, x}\left[\int_{s}^{T_{R}} \sum_{i=1}^{n} \frac{\partial \phi}{\partial x_{i}} \cdot \mathrm{d} \gamma_{i}^{c}+\sum_{s \leqslant t_{k} \leqslant T_{R}} \sum_{i=1}^{n} \frac{\partial \phi}{\partial x_{i}}\left(t_{k}, \hat{X}_{(k)}^{\gamma}\right) \Delta \gamma_{i}\left(t_{k}\right)\right] .
$$

So condition (2.6) gives

$$
\begin{aligned}
\phi(s, x) & \geqslant \mathrm{E}^{s, x}\left[\int_{s}^{T_{R}} \sum_{i=1}^{n} \frac{\partial \phi}{\partial x_{i}} \mathrm{~d} \gamma_{i}^{c}+\sum_{i=1}^{n} \sum_{s \leqslant t_{k} \leqslant T_{R}} f_{i}\left(t_{k}\right) \Delta \gamma_{i}\left(t_{k}\right)\right]+\mathrm{E}^{s, x}\left[\phi\left(T_{R}, X^{\gamma}\left(T_{R}\right)\right)\right] \\
& \geqslant \mathrm{E}^{s, x}\left[\int_{s}^{T_{R}} f \cdot \mathrm{d} \gamma\right]+\mathrm{E}^{s, x}\left[\phi\left(T_{R}, X^{\gamma}\left(T_{R}\right)\right)\right] .
\end{aligned}
$$

Since $R<\infty, \gamma \in \Gamma$ were arbitrary and $\phi \geqslant 0$, this proves that (2.8) holds, that is,

$$
\phi(s, x) \geqslant \Phi(s, x) \text {. }
$$

(ii) Next, assume that $D$ is given by (2.9) and that (2.10)-(2.13) hold. Then the above calculations with $\gamma$ replaced by $\hat{\gamma}$ give equality everywhere and we end up with equality in (2.15). Hence

$$
\phi(s, x)=\mathrm{E}^{s, x}\left[\int_{s}^{T_{R}} f \cdot \mathrm{d} \hat{\gamma}\right]+\mathrm{E}^{s, x}\left[\phi\left(T_{R}, X^{\hat{\gamma}}\left(T_{R}\right)\right)\right] .
$$

By letting $R \rightarrow \infty$ and using (2.14), we obtain 


$$
\phi(s, x)=\mathrm{E}^{s, x}\left[\int_{s}^{T} f \cdot \mathrm{d} \hat{\gamma}\right] .
$$

Combining this with (2.8), we see that

$$
\phi(s, x)=\Phi(s, x) \quad \text { and } \hat{\gamma} \text { is optimal. }
$$

To illustrate the content of this theorem a typical (generic) situation in the case $n=2$ is shown in Figure 1 . Here the survival set is $S=\mathbb{R} \times(0, \infty) \times(0, \infty)$. The non-intervention region $D$ is bounded by the two curves $\Lambda_{1}, \Lambda_{2}$ given by

$$
\Lambda_{i}=\left\{\left(x_{1}, x_{2}\right) ; \frac{\partial \phi}{\partial x_{i}}\left(t, x_{1}, x_{2}\right)=f_{i}(t)\right\}, \quad i=1,2
$$

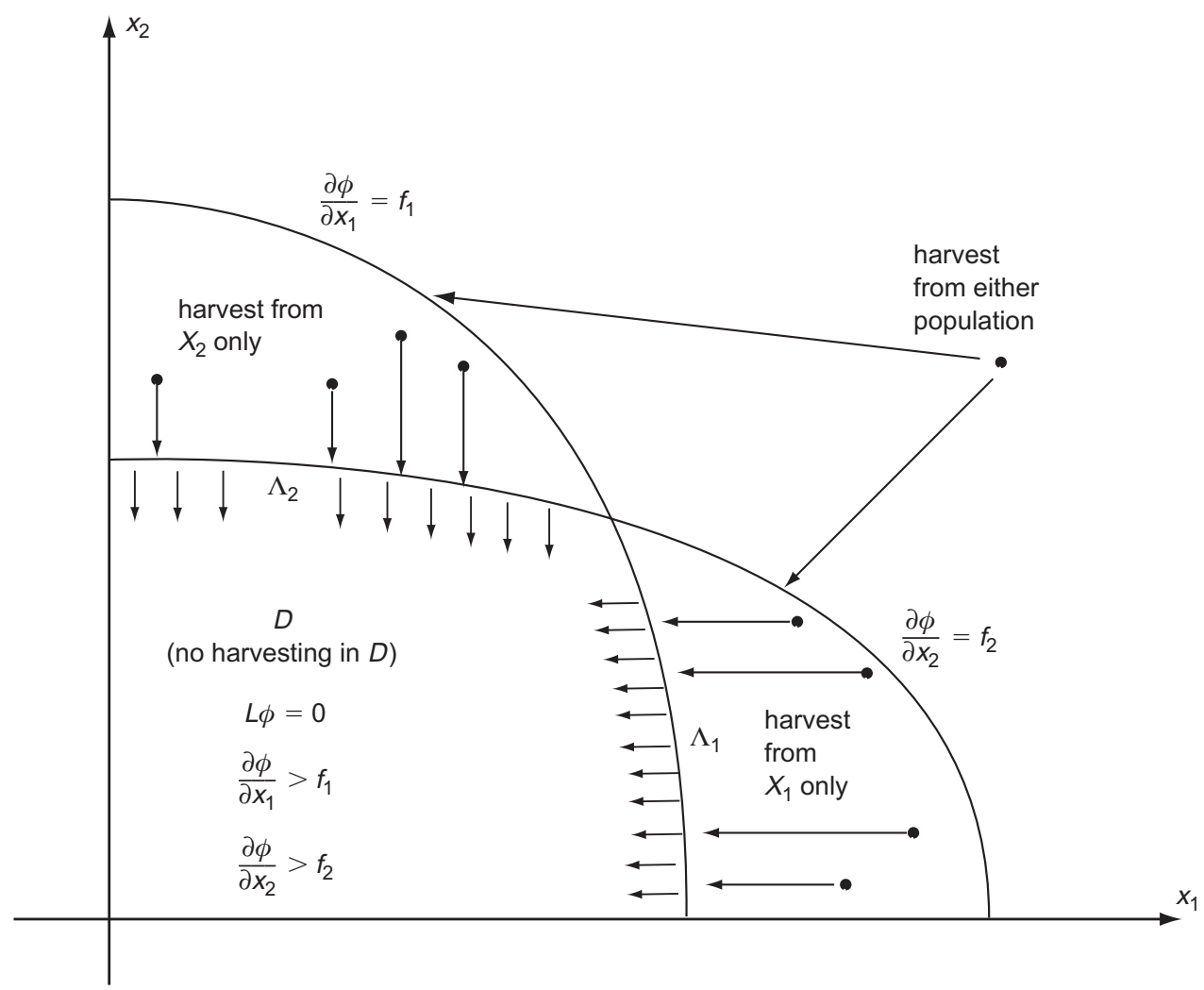

Figure 1. The one-at-a-time principle: harvest only from population 1 (horizontal reflection) on the curve $\Lambda_{1} \cap \partial D$; harvest only from population 2 (vertical reflection) on the curve $\Lambda_{2} \cap \partial D$. 
(fixing the time $t$ ). From (2.12) we see that $\mathrm{d} \hat{\gamma}_{2}=0$ on $\Lambda_{1}$, while $\mathrm{d} \hat{\gamma}_{1}=0$ on $\Lambda_{2}$. Note that generically (e.g. when $\sigma \sigma^{\mathrm{T}}$ is bounded away from 0$)$ the probability that $\left(X_{1}(t), X_{2}(t)\right)$ hits the intersection point $\Lambda_{1} \cap \Lambda_{2}$ is 0 . A similar argument holds in any dimension $n$. Then generically $\Lambda_{i}$ will be submanifolds of dimension $n-1$ and the intersection of two or several $\Lambda_{i}$ will have dimension $n-2$ or lower, and if, for example, the eigenvalues of $\sigma \sigma^{\mathrm{T}}$ are bounded away from 0 the diffusion will almost surely never hit such sets. Thus we arrive heuristically - at the following principle:

Principle 2.1 (One-at-a-time principle). Generically it is almost surely never optimal to harvest from more than one population at a time.

We conjecture that, if properly formulated, a result like this can be rigorously proved.

Remark. Suppose we have found a function $\phi$ satisfying conditions (2.6)-(2.10). Then a harvesting strategy $\hat{\gamma}$ satisfying (2.11)-(2.13) can (usually) be found by solving the Skorohod stochastic differential equation for the reflection $Y^{\hat{\gamma}}(t)$ of the process $Y(t)$ in $D$. This means that we find a harvesting strategy $\hat{\gamma}$ with the following properties. If we define $Y^{\hat{\gamma}}(t)$ by the equation

$$
\mathrm{d} Y^{\hat{\gamma}}(t)=b\left(Y^{\hat{\gamma}}(t)\right) \mathrm{d} t+\sigma\left(Y^{\hat{\gamma}}(t)\right) \mathrm{d} B(t)-\theta\left(Y^{\hat{\gamma}}(t)\right) \mathrm{d} \hat{\gamma}(t),
$$

where $\theta(y)$ is the $n \times n$ diagonal matrix with $i$ th diagonal entry

$$
\theta_{i i}(y)=\frac{\partial \phi}{\partial x_{i}}(y)-f_{i}(t), \quad 1 \leqslant i \leqslant n, y=(t, x),
$$

then

$$
Y^{\hat{\gamma}}(t) \in \bar{D}, \quad \text { for all } t>s,
$$

where $\bar{D}$ is the closure of $D$, and

$$
\theta\left(Y^{\hat{\gamma}}(t)\right) \cdot \mathrm{d} \hat{\gamma}(t)=0 \quad \text { if } Y^{\hat{\gamma}}(t) \notin \partial D .
$$

If such a $\hat{\gamma}$ exists, it is called the local time for $Y(t)$ in the direction $\theta$ at $\partial D$.

For more information about Skorohod stochastic differential equations, sufficient conditions for the existence of a solution, and so on, see Bass (1997), Freidlin (1985) and Lions and Sznitman (1984).

Remark. Theorem 2.1 is a verification theorem. It gives sufficient conditions that a given function actually coincides with the value function $\Phi$ and, if it does, the theorem tells us how to find an optimal control. It is natural to ask if the converse is true: does the value function $\Phi$ always satisfy the conditions of Theorem 2.1? If we concentrate on the three most basic variational inequalities, (2.6), (2.7) and (2.10), we see that these can be combined into one equation,

$$
\max \left\{L \phi(t, x), \max _{1 \leqslant i \leqslant n}\left\{f_{i}(t)-\frac{\partial \phi}{\partial x_{i}}(t, x)\right\}\right\}=0 \quad \text { in } S^{0}
$$


Then the question is: does $\Phi$ always satisfy (2.22)? The answer is no in general, simply because $\Phi$ need not be smooth enough for $L \Phi$ to make (strong) sense. However, if we interprete (2.22) in the appropriate weak sense of viscosity, then the answer is yes, at least if $b$ and $\sigma$ are bounded functions. This was proved in a general setting by Haussmann and Suo (1995a, Theorem 5.5): if $b$ and $\sigma$ are bounded then indeed $\Phi$ is the unique viscosity solution of (2.22). This result, albeit important, does not eliminate the need for Theorem 2.1. First, the assumption that $b$ and $\sigma$ are bounded is too strict for many applications. Second, Theorem 2.1 also provides a method of finding an optimal control, not just the value function.

\section{Applications}

In this section we apply Theorem 2.1 to study a specific case. Unfortunately, it seems difficult to find multidimensional examples which can be solved explicitly. Our example (with $n=2$ ) has the advantage of being solvable, but the disadvantage of being slightly degenerate, in the sense that the two harvesting lines $\Lambda_{1}, \Lambda_{2}$ defined in (2.17) coincide. Because of this degeneracy this example is actually a counterexample to the one-at-a-time principle. Nevertheless, we include it because it serves as a good illustration of how to apply Theorem 2.1 in specific cases. The example is in Schulstok (1998), and presented here with his kind permission. He also considers the $n$-dimensional analogue. The example may be regarded as a two-dimensional analogue of the example studied in Jeanblanc-Picqué and Shiryaev (1995).

Example 3.1 Optimization of the flow of dividends.

In this example it is more natural to use a financial interpretation than a biological interpretation of the optimal harvesting problem. Consider a market with two investments whose values $X_{1}(t), X_{2}(t)$ at time $t$ are given by the equations

$$
\mathrm{d} X_{i}(t)=b_{i} \mathrm{~d} t+\sigma_{i 1} \mathrm{~d} B_{1}(t)+\sigma_{i 2} \mathrm{~d} B_{2}(t), \quad i=1,2,
$$

where $b_{i}$ and $\sigma_{i j}$ are constants.

Suppose each unit of $X_{i}$ has a constant price $\theta_{i}$. If we pay out dividends (i.e. apply a harvesting strategy) $\gamma(t, \omega)=\left(\gamma_{1}(t, \omega), \gamma_{2}(t, \omega)\right)$ then the total value $X_{i}^{\gamma}(t)$ of the investments will satisfy the equation

$$
\begin{aligned}
& \mathrm{d} X_{i}^{\gamma}(t)=b_{i} \mathrm{~d} t+\sigma_{i 1} \mathrm{~d} B_{1}(t)+\sigma_{i 2} \mathrm{~d} B_{2}(t)-\mathrm{d} \gamma_{i}(t), \quad t \geqslant 0, \\
& X_{i}^{\gamma}\left(0^{-}\right)=x_{i} .
\end{aligned}
$$

The corresponding expected total discounted payoff is in this case given by

$$
J^{\gamma}\left(s, x_{1}, x_{2}\right)=\mathrm{E}^{x_{1}, x_{2}}\left[\int_{0}^{T^{-}} \mathrm{e}^{-\rho(s+t)}\left(\theta_{1} \mathrm{~d} \gamma_{1}(t)+\theta_{2} \mathrm{~d} \gamma_{2}(t)\right)\right],
$$

where

$$
T=\inf \left\{t>0 ; \theta_{1} X_{1}(t)+\theta_{2} X_{2}(t) \notin S\right\}
$$

is the time of bankruptcy, with solvency region $S=\left\{\left(x_{1}, x_{2}\right) ; \theta_{1} x_{1}+\theta_{2} x_{2} \geqslant 0\right\}$. 
We seek an optimal divident payment policy $\gamma^{*}$ and the value function $\Phi$ given by

$$
\Phi\left(s, x_{1}, x_{2}\right)=\sup _{\gamma} J^{\gamma}\left(s, x_{1}, x_{2}\right)=J^{\gamma^{*}}\left(s, x_{1}, x_{2}\right) .
$$

From (3.4) we see that it is natural to try to look for a function $\Phi$ of the form

$$
\Phi\left(s, x_{1}, x_{2}\right)=\mathrm{e}^{-\rho s} \Psi\left(x_{1}, x_{2}\right) .
$$

Hence in Theorem 2.1 we put

$$
\phi\left(s, x_{1}, x_{2}\right)=\mathrm{e}^{-\rho s} \psi\left(x_{1}, x_{2}\right) .
$$

In terms of $\psi$, the inequalities (2.6), (2.7) take the following form in $S$ :

$$
\frac{\partial \psi}{\partial x_{i}} \geqslant \theta_{i}, \quad i=1,2
$$

and

$$
L_{0} \psi\left(x_{1}, x_{2}\right):=-\rho \psi+\sum_{i=1}^{2} b_{i} \frac{\partial \psi}{\partial x_{i}}+\frac{1}{2} \sigma_{1}^{2} \frac{\partial^{2} \psi}{\partial x_{1}^{2}}+\sigma_{1} \cdot \sigma_{2} \frac{\partial^{2} \psi}{\partial x_{1} \partial x_{2}}+\frac{1}{2} \sigma_{2}^{2} \frac{\partial^{2} \psi}{\partial x_{2}^{2}} \leqslant 0,
$$

where $\sigma_{i}=\left(\sigma_{i 1}, \sigma_{i 2}\right)$ and $\sigma_{i} \cdot \sigma_{j}$ is the dot product of $\sigma_{i}$ and $\sigma_{j}, 1 \leqslant i, j \leqslant 2$.

Now try a function $\psi$ of the form

$$
\psi\left(x_{1}, x_{2}\right)=F(z), \quad \text { where } z=\theta_{1} x_{1}+\theta_{2} x_{2} .
$$

Then (3.7) leads to just one inequality, namely

$$
F^{\prime}(z) \geqslant 1, \quad \text { for } z>0,
$$

and (3.8) takes the form

$$
A F(z):=-\rho F(z)+\alpha F^{\prime}(z)+\beta F^{\prime \prime}(z) \leqslant 0, \quad \text { for } z>0,
$$

where

$$
\begin{aligned}
& \alpha=\theta_{1} b_{1}+\theta_{2} b_{2}, \\
& \beta=\frac{1}{2} \sigma_{1}^{2} \theta_{1}^{2}+\sigma_{1} \cdot \sigma_{2} \theta_{1} \theta_{2}+\frac{1}{2} \sigma_{2}^{2} \theta_{2}^{2} .
\end{aligned}
$$

The general solution of $A F=0$ is

$$
F(z)=C_{1} \mathrm{e}^{r_{1} z}+C_{2} \mathrm{e}^{r_{2} z},
$$

where $C_{1}, C_{2}$ are arbitrary constants and

$$
r_{1}=\frac{1}{2 \beta}\left(-\alpha+\sqrt{\alpha^{2}+4 \rho \beta}\right), \quad r_{2}=\frac{1}{2 \beta}\left(-\alpha-\sqrt{\alpha^{2}+4 \rho \beta}\right) .
$$

We now guess that the non-intervention region $D$ has the form

$$
D=\left\{z ; 0<z<z^{*}\right\}
$$

for some $z^{*}>0$. Thus, by (2.9) and (2.10) it is natural to try to put 


$$
F(z)= \begin{cases}z+K & \text { for } z \geqslant z^{*}, \\ C_{1} \mathrm{e}^{r_{1} z}+C_{2} \mathrm{e}^{r_{2} z} & \text { for } 0<z<z^{*},\end{cases}
$$

for some constant $K$, and then try to determine the parameters $C_{1}, C_{2}, K$ and $z^{*}$ such that $F$ becomes a $C^{2}$ function at $z=z^{*}$.

Continuity at $z=z^{*}$ gives the equation

$$
C_{1} \mathrm{e}^{r_{1} z^{*}}+C_{2} \mathrm{e}^{r_{2} z^{*}}=z^{*}+K .
$$

Differentiability at $z=z^{*}$ gives the equation

$$
r_{1} C_{1} \mathrm{e}^{r_{1} z^{*}}+r_{2} C_{2} \mathrm{e}^{r_{2} z^{*}}=1 .
$$

Twice differentiability at $z=z^{*}$ gives the equation

$$
r_{1}^{2} C_{1} \mathrm{e}^{r_{1} z^{*}}+r_{2}^{2} C_{2} \mathrm{e}^{r_{2} z^{*}}=0 .
$$

In addition, we know that $F(0)=0$, hence

$$
C_{1}+C_{2}=0, \quad \text { i.e. } C_{1}=-C_{2}=: C \text {. }
$$

The four equations (3.18)-(3.21) determine $C_{1}, C_{2}, C_{3}$ and $z^{*}$ uniquely: from (3.20) and (3.21) we obtain

$$
z^{*}=\frac{2 \ln \left|r_{2} / r_{1}\right|}{r_{1}-r_{2}}>0
$$

and this gives, by (3.19) and (3.21),

$$
C=\left(r_{1} \mathrm{e}^{r_{1} z^{*}}-r_{2} \mathrm{e}^{r_{2} z^{*}}\right)^{-1} .
$$

Finally, applying this to (3.18), we obtain

$$
K=C\left(\mathrm{e}^{r_{1} z^{*}}-\mathrm{e}^{r_{2} z^{*}}\right)-z^{*} .
$$

With this choice of $C_{1}=C, C_{2}=-C, K$ and $z^{*}$, all the conditions of Theorem 2.1 are satisfied and we conclude that the value function $\Phi$ is given by

$$
\Phi\left(s, x_{1}, x_{2}\right)= \begin{cases}C \mathrm{e}^{-\rho s}\left(\mathrm{e}^{r_{1} z}-\mathrm{e}^{r_{2} z}\right) & \text { for } 0 \leqslant z<z^{*}, \\ \mathrm{e}^{-\rho s}(z+K) & \text { for } z^{*} \leqslant z,\end{cases}
$$

with $z=\theta_{1} x_{1}+\theta_{2} x_{2}$.

Thus we obtain that the optimal strategy is to do nothing as long as $\left(X_{1}(t), X_{2}(t)\right) \in D$, that is, as long as

$$
0<\theta_{1} X_{1}(t)+\theta_{2} X_{2}(t)<z^{*}
$$

(see Figure 2). Then when $\left(X_{1}(t), X_{2}(t)\right)$ reaches the value $z^{*}$, we harvest (pay dividends) according to local time $\gamma^{*}=\hat{\gamma}$ at $\partial D$ of the reflected diffusion $\left(X_{1}^{\hat{\gamma}}(t), X_{2}^{\hat{\gamma}}(t)\right)$, reflected in the direction $-\left(\theta_{1}, \theta_{2}\right)$. Intuitively this means that we pay exactly what is needed to keep $\left(X_{1}^{\hat{\gamma}}(t), X_{2}^{\hat{\gamma}}(t)\right)$ to the left of the line $\theta_{1} x_{1}+\theta_{2} x_{2}=z^{*}$. Moreover, we harvest from $X_{1}$ and $X_{2}$ simultaneously, at the ratio 


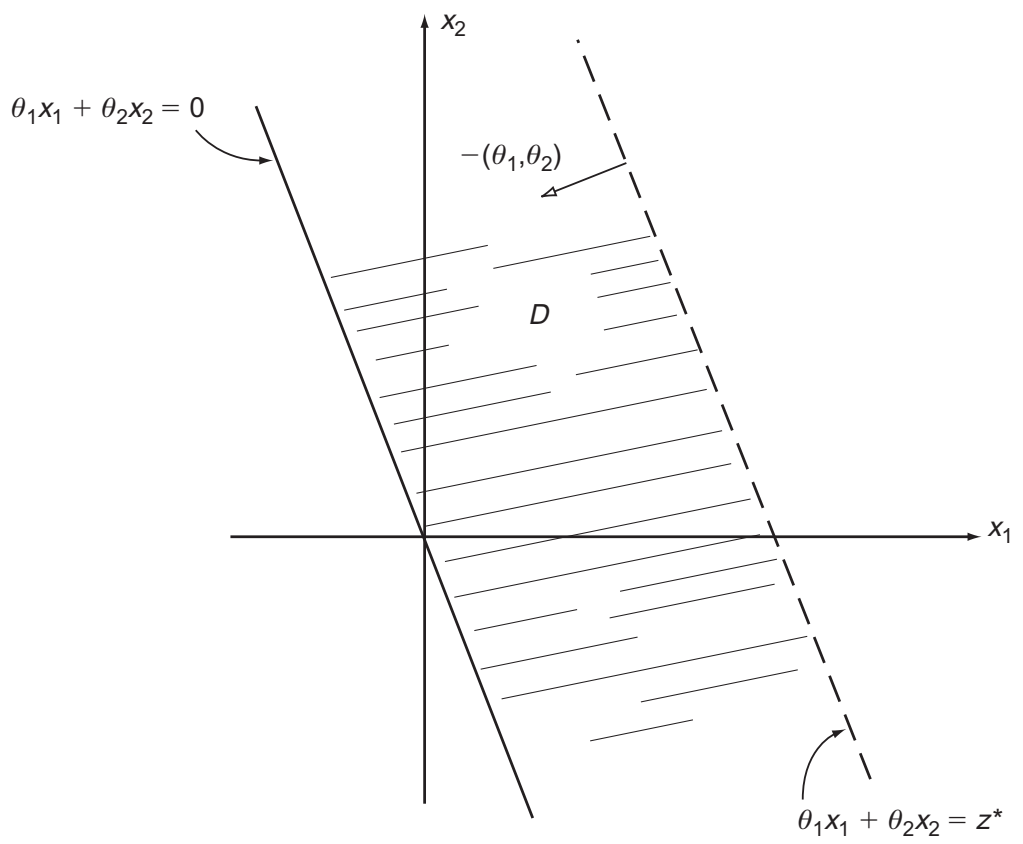

Figure 2. Non-intervention region $D$ in a market with two investments. To the right of $D$, dividends are paid.

$$
\frac{\Delta x_{2}}{\Delta x_{1}}=\frac{\theta_{2}}{\theta_{1}}
$$

Remark. The one-dimensional nature of the solution of this problem makes it natural to ask whether the whole problem could be solved by transforming it to a one-dimensional problem from the beginning. One could try to put

$$
Z(t)=\theta_{1} X_{1}(t)+\theta_{2} X_{2}(t)
$$

and then ask for the optimal dividend policy for $Z(t)$, that is, try to find $H$ and $\Gamma^{*}$ such that

$$
H(s, z)=\sup _{\Gamma} \mathrm{E}^{z}\left[\int_{0}^{T} \mathrm{e}^{-\rho(s+t)} \mathrm{d} \Gamma(t)\right]=\mathrm{E}^{z}\left[\int_{0}^{T} \mathrm{e}^{-\rho(s+t)} \mathrm{d}^{*}(t)\right],
$$

the supremum being taken over all harvesting strategies $\Gamma(t)$ for $Z(t)$. The calculation above shows that this problem has the same value function as problem (3.6). More precisely,

$$
\Phi\left(s, x_{1}, x_{2}\right)=H\left(s, \theta_{1} x_{1}+\theta_{2} x_{2}\right) .
$$

This identity is not obvious, because it is required for the harvesting strategies $\Gamma(t)$ for $Z(t)$ that they are adapted to the filtration $\mathscr{G}_{t}$ generated by $\{Z(r) ; r \leqslant t\}$. This filtration is strictly smaller than the filtration $\mathscr{F}_{t}$ generated by $\left\{X_{i}(r) ; r \leqslant t, i=1,2\right\}$. 


\section{Acknowledgements}

We wish to thank Luis Alvarez, Fred Espen Benth, Trond Myhre, Kristin Reikvam, Uwe Roesler and Bjørn Schulstok for helpful comments.

\section{References}

Alvarez, L. (2000) Singular stochastic control in the presence of a state-dependent yield structure. Stochastic Process. Appl., 86, 323-343.

Alvarez, L., Lungu, E. and Øksendal, B. (2001) Optimal multi-dimensional stochastic harvesting with density-dependent prices. Preprint, University of Oslo.

Alvarez, L. and Shepp, L. (1998) Optimal harvesting of stochastically fluctuating populations. J. Math. Biol., 37, 155-177.

Bass, R. (1997) Diffusions and Elliptic Operators. New York: Springer-Verlag.

Braumann, C.A. (2000) Variable effort fishing models in random environments. Math. Biosci., 156, $1-19$.

Benth, F.E. and Reikvam, K. (1998) On a connection between singular stochastic control and optimal stopping. Research Report 1999-28, MaPhySto.

Freidlin, M. (1985) Functional Integration and Partial Differential Equations. Princeton, NJ: Princeton University Press.

Gause, G.F. and Witt, A.A. (1935) Behaviour of mixed populations and the problem of natural selection. Amer. Naturalist, 69, 596-609.

Haussmann, U. and Suo, W. (1995a) Singular optimal stochastic controls I: Existence. SIAM J. Control Optim., 33, 916-936.

Haussmann, U. and Suo, W. (1995b) Singular optimal stochastic controls II: Dynamic programming. SIAM J. Control Optim., 33, 937-959.

Jeanblanc-Picqué, M. and Shiryaev, A. (1995) Optimization of the flow of dividends. Russian Math. Surveys, 50, 257-277.

Lungu, E. and Øksendal, B. (1997) Optimal harvesting from a population in a stochastic crowded environment. Math. Biosci., 145, 47-75.

Lions, P.L. and Sznitman, A.S. (1984) Stochastic differential equations with reflecting boundary conditions. Comm. Pure Appl. Math., 37, 511-537.

Myhre, T. (1998) Connections between optimal stopping and singular control: An approach based on variational inequalities. Research report no. 395, University of Aarhus.

Protter, P. (1990) Stochastic Integration and Differential Equations. Berlin: Springer-Verlag.

Schulstok, B. (1998) Optimal income by harvesting under uncertainty. Cand. Scient. thesis, University of Oslo.

Received September 1999 and revised February 2001 